\title{
Synthesis, Structural Order and Magnetic Behavior of Self-Assembled $\varepsilon$-Co Nanocrystal Arrays
}

\author{
Victor F. Puntes and Kannan M. Krishnan
}

\begin{abstract}
The synthesis of magnetic nanoparticles with monodispere size distributions, their self assembly into ordered arrays and their magnetic behavior as a function of structural order (ferrofluids and 2D assemblies) are presented. Magnetic colloids of monodispersed, passivated, cobalt nanocrystals were produced by the rapid pyrolysis of cobalt carbonyl in solution. The size, size distribution (std. dev. $<5 \%$ ) and the shape of the nanocrystals were controlled by varying the surfactant, its concentration, the reaction rate and the reaction temperature. The Co particles are defect-free single crystals with a complex cubic structure related to the beta phase of manganese $(\varepsilon-\mathrm{Co})$. In the 2D assembly, a collective behavior was observed in the low-field susceptibility measurements where the magnetization of the zero field cooled process increases steadily and the magnetization of the field cooling process is independent the temperature. This was different from the observed behavior in a sample comprised of disordered interacting particles. A strong paramagnetic contribution appears at very low temperatures where the magnetization increases drastically after field cooling the sample. This has been attributed to the Co surfactant-particle interface since no magnetic atomic impurities are present in these samples.
\end{abstract}

Index Terms-Chemical synthesis of ferrofluids, Co nanocrystals, finite size effects, self assembly.

\section{INTRODUCTION}

$\mathbf{N}$ ANOSCALE and nanoparticle materials are expected to exhibit unusual chemical and physical properties different from those of either the bulk materials or single atoms [1], [2]. Properties such as ferro- or ferri-magnetism as well as superconductivity are not intrinsic to a molecule or an ion but arise from a cooperative solid-state (bulk) behavior. Such properties are a consequence of the interactions between the molecules or ions and hence, methods to predict, control and modulate the solid state structure are essential to the understanding and manipulation of such behavior.

Studies of the magnetic properties of fine particles have been complicated by the difficulties arising from their dispersions in size, shape, surface defects and poor crystallinity. Thus, development of new methods for the preparation of monodispersed magnetic particles is crucial to deconvolute finite-size, interactions and surface effects. In addition, conventional magnetic storage technology is rapidly approaching its scaling limit [3]. Thin granular films of sputtered ferromagnetic nanocrystals are the basis of conventional magnetic storage (hard drives) devices.

Manuscript received October 13, 2000.

This work was supported in part by the U.S. Department of Energy, Basic Energy Sciences under Grant DE-ACO3-SF00096.

V. F. Puntes and K. M. Krishnan are with the Materials Sciences Division, Lawrence Berkeley National Laboratory, University of California, Berkeley, CA 94720 (e-mail: krishnan@lbl.gov).

Publisher Item Identifier S 0018-9464(01)06523-2.
Progress in magnetic recording density is due, in part, to the development of media with increasingly finer grain sizes. However, to have a sufficient signal to noise ratio in the recorded signal, the number of grains needed to store a bit is about thousand grains [3]. The study of magnetic nanoparticles is thus of both fundamental and pressing technological interest as the grain size of the advanced recording media is rapidly shrinking toward the superparamagnetic size (about $10 \mathrm{~nm}$ for hcp Co) limit.

Different magnetic nanoparticles, such as Co [4] and FePt [5], have been recently synthesized by chemical reduction methods. Other methods include the reverse micelle technique for the synthesis of Co particles [6]. Those particles may display a twin crystal structure and chemical contamination from the solvent, the reducing agent or the precursor. In addition, a size selective precipitation is often required in order to obtain a narrow $(<10 \%)$ size dispersion, which also seems to be the prerequisite to obtain 2D self-assemblies [7].

\section{SYNTHESIS AND STRUCTURE}

Rapid injection of organometallic reagents in a hot coordinating solvent produces a temporally discrete homogeneous nucleation and has been mainly employed for the production of monodisperse, passivated, semiconductor nanocrystals [8]. Following this approach, in this paper we present a new method for the production of magnetic colloids (ferrofluids) of stabilized cobalt nanocrystals based on the rapid pyrolysis of the organic precursor $\mathrm{Co}_{2}(\mathrm{CO})_{8}$ in an inert ( $\mathrm{Ar}$ ) atmosphere and in the presence of an organic surfactant (oleic acid and trioctylphosphonic acid) at high temperatures. These conditions lead to extremely narrow size distributions. The size and shape of the Co particles are controlled via the surfactant, its concentration and temperature of the reaction. The surfactant has the ability to control the size and shape of the synthesized nanocrystals by tuning the surface tension of the growing particle. In addition, it prevents their agglomeration, passivates them against oxidation and determines the interparticle distance in the dried samples; however, the surfactant may also affect the uniformity or magnitude of the magnetization due to quenching of surface moments. Colloidal solutions of Co nanocrystals are stable over months and no evidence for the formation of $\mathrm{CoO}$ or $\mathrm{Co}_{3} \mathrm{O}_{4}$ has been observed.

Passivated Co nanocrystal particles with well defined shapes, i.e., spheres (3 to $17 \mathrm{~nm}$ ), cubes and rods (Fig. 1) with nearly monodisperse (std. dev. $<5 \%$ ) size have been produced by the injection of a saturated solution of $\mathrm{Co}_{2}(\mathrm{CO})_{8}$ in o-Dichlorobenzene $(3 \mathrm{ml})$ in an o-dichlorobenzene hot bath 

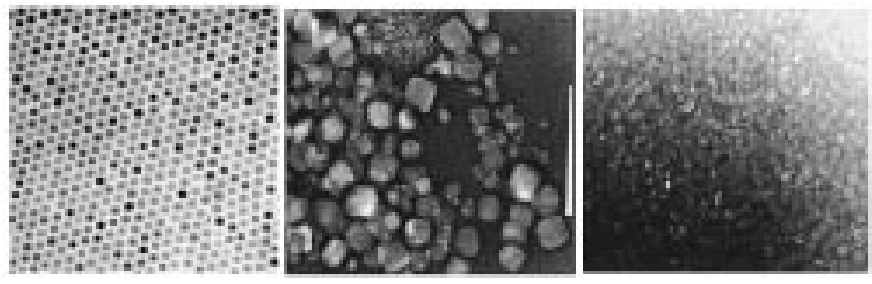

Fig. 1. BF TEM images of $10 \mathrm{~nm}$ spherical Co particles, $25 \mathrm{~nm}$ Co cubes and $4: 4: 10 \mathrm{~nm}$ Co rods, respectively.

(a)

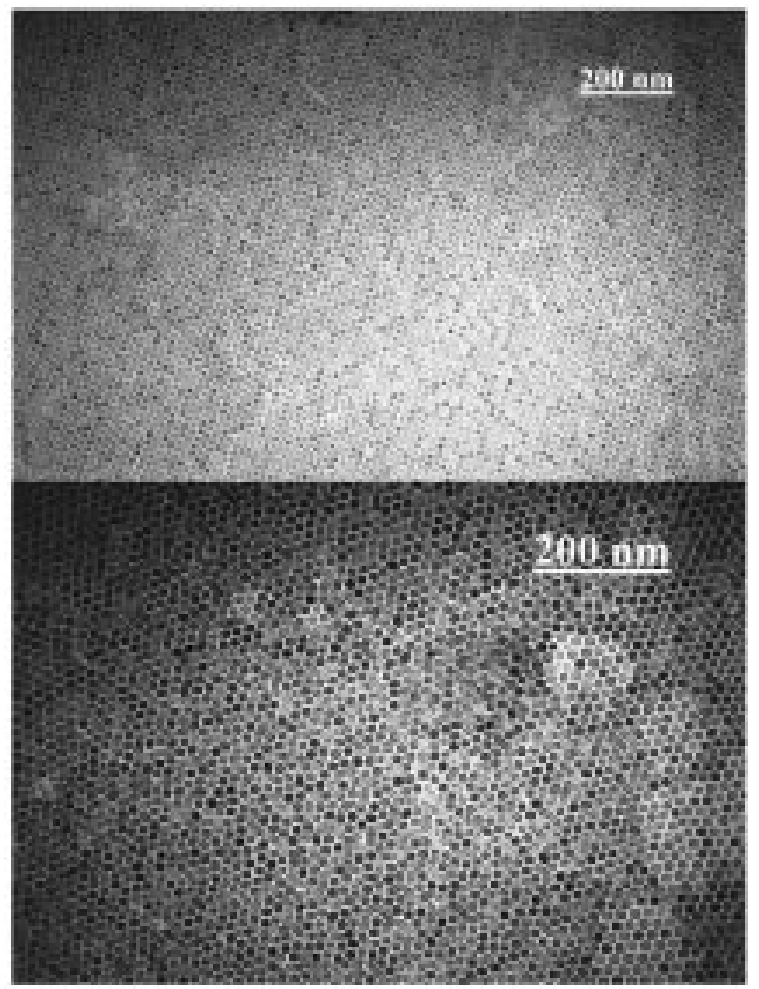

Fig. 2. BF TEM image of $10 \mathrm{~nm}$ spherical Co particles. (a) First monolayer. (b) First two monolayers or a bilayer.

$(12 \mathrm{ml})$. The surfactant is present in the hot bath at concentrations of about $1 \%$.

In this paper we will discuss the magnetic properties of the spherical particles and their arrays by self assembly. XRD patterns [9] showed that the particles are $\varepsilon$-Co single-crystals, presenting a complex cubic structure related to the beta phase of the manganese [10].

These particles have been observed to produce 2D self-assemblies [Fig. 2(a)] when evaporated at low rates in a controlled atmosphere, provided that the particle size distribution is narrow enough and the initial concentration is appropriate. Thus, larger concentrations will produce 3D self-assemblies. The assembly is determined by the size of the crystals and the thickness of the coating layer, which generally is about $2 \mathrm{~nm}$. Hexagonal arrangement of the particles is observed in these self-assemblies. Large areas of regular hexagonal assemblies have not been observed in the larger particles (above the superparamagnetic-ferromagnetic transition, i.e., $\sim 12 \mathrm{~nm}$ ) due to the strong dipole-dipole interactions during agglomeration. Higher initial concentrations lead to 3D self
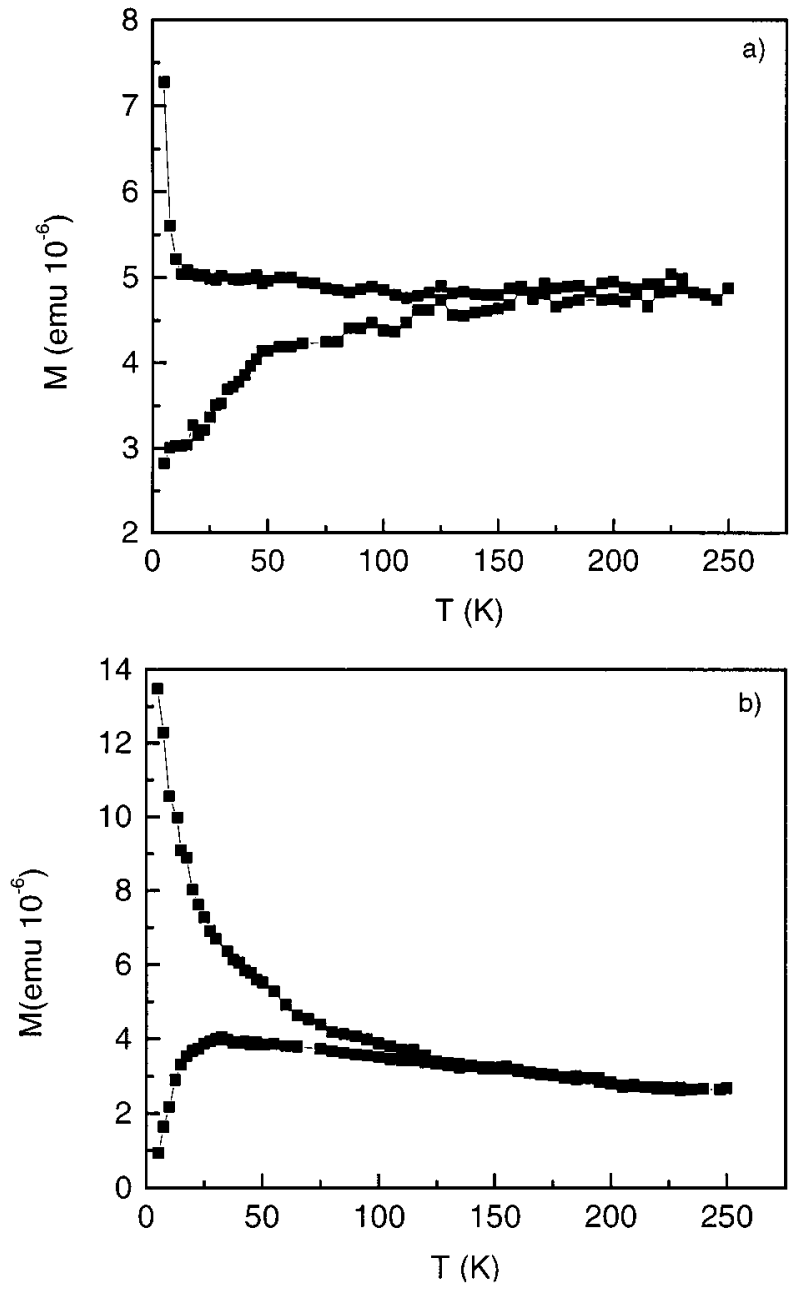

Fig. 3. ZFC/FC curves measured at 50 Oe. (a) An ordered 2D self assembly of $10 \mathrm{~nm} \varepsilon$-Co crystals. (b) Disordered agglomeration of $10 \mathrm{~nm} \varepsilon$-Co crystals.

assemblies where the particles in the second layer occupy sites determined by close-packing arrangements [Fig. 2(b)].

Microstructural analysis using characteristic $\mathrm{x}$-rays or fine structure in electron energy-loss spectroscopies in a TEM with a field emission source and operated in the fine probe $(<10 \mathrm{~nm})$ mode did not show any significant evidence for the presence of $\mathrm{CoO}$ in the particles surface.

\section{MAGNeTIC BeHAVIOR}

Magnetic properties of those nanoparticles have been studied from zero-field cool and field cool (ZFC/FC) magnetization curves and from hysteresis loops at different temperatures. $\varepsilon$-Co is a soft magnetic material that displays a magnetocrystalline anisotropy between that of the fcc and hcp structures. From the hysteresis loops the anisotropy has been estimated to be $\sim 1.5 \times 10^{6} \mathrm{erg} / \mathrm{cm}^{3}$ at room temperature [11]. For both hcp and fcc $\mathrm{Co}$, the particles are expected to be single domains [12] at sizes smaller than $70 \mathrm{~nm}$; thus we expect a similar limit in $\varepsilon$-Co which has a comparable anisotropy.

The ZFC/FC curves of a 2D nanocrystal array [Fig. 3(a)] ere recorded at 50 Oe. The measured sample is the TEM grid imaged in Fig. 1 with over an 80\% of 2D self assembly coverage. The ZFC shows a gradual increase of the magnetization up to 


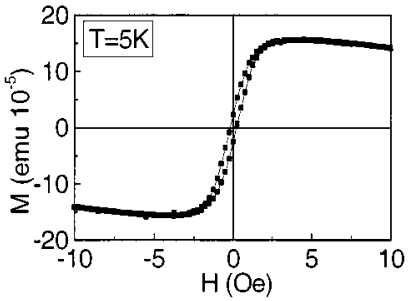

(a)

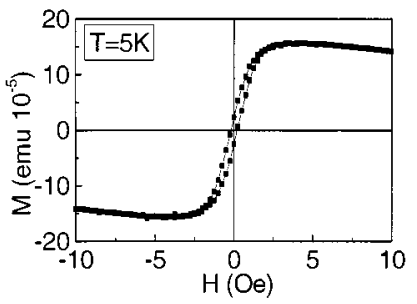

(c)

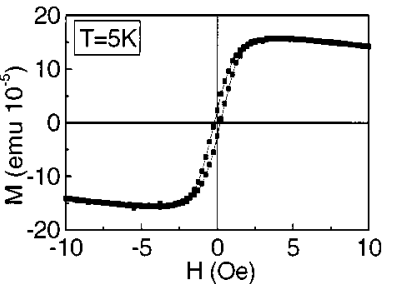

(b)

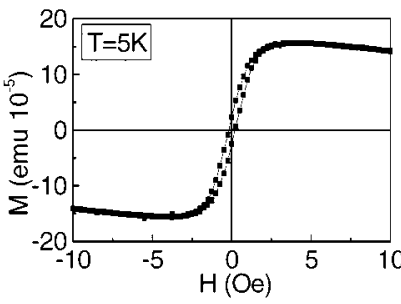

(d)
Fig. 4. Hysteresis loops of: in-plane magnetization of a 2D self assembly of $10 \mathrm{~nm}$ Co particles. (a) $5 \mathrm{~K}$. (b) $275 \mathrm{~K}$. Hysteresis loop of the dried powder. (c) $5 \mathrm{~nm}$ Co spherical particles. (d) $9 \mathrm{~nm}$ Co spherical particles.

around $47 \mathrm{~K}$ which suggest a progressive rotation of the magnetization of blocked magnetic particles toward the field direction. At $47 \mathrm{~K}$ the magnetization, instead of forming a peak as expected for small particles [12], steadily increases up to RT. Between 47 and $300 \mathrm{~K}$ the $\mathrm{FC}$ curve is completely flat, all in all suggesting a ferromagnet-like behavior. This could be explained by considering that dipolar interactions in highly-ordered particles systems could be magnetizing [13], while in more disordered systems dipolar interactions are generally demagnetizing [14].

In the $\mathrm{ZFC} / \mathrm{FC}$ curve of a disordered sample [Fig. 3(b)], we observe the expected behavior for a collection of interacting particles, with a broad maximum in the ZFC curve. The samples were prepared by dispersing the dried particles in oleic acid which solidifies at $16^{\circ} \mathrm{C}$. The peak is around $40 \mathrm{~K}$, which is larger than that expected for a noninteracting sample, due to the effect of interactions shifting the energy barrier distribution to higher values. Interactions are able to overcome the thermal decay and the superparamagnetic state is not reached below room temperature. The strong increase in the FC curve at low $T$ is probably due to the fact that some particles aligned themselves to the applied field at high $T(250-300 \mathrm{~K})$ whilst the oleic acid was viscous. A strong paramagnetic contribution has been observed in the FC curve at very low temperatures where the magnetization increases drastically after field-cooling the sample. This has been attributed to the Co-surfactant particle interface since no magnetic atomic impurities are present in these samples.

A small hysteresis is observed at low $T$ and it disappears at larger $T$ [Fig. 4(a) and (b)] where the thermal energy progressively overcomes the interactions. At high fields $(H>2000 \mathrm{Oe})$ the diamagnetic contribution of the substrate is observed in the negative slope of the magnetization curve. Paramagnetic effects in the 2D array appear at very low temperature, thus they are not observed in the hysteresis loops.

The hysteresis loops at room temperature of the dried powder for two different particle sizes is shown in Fig. 4(c) and (d). The smaller Co spheres $(5 \mathrm{~nm})$ dried sample is more difficult to magnetize (saturation field larger) than the larger $(9 \mathrm{~nm})$ ones. The former also displays a larger superparamagnetic contribution at high fields and a lower magnetization, both probably related to finite-size and surface effects which increase with decreasing particle size [15]. In agglomerated samples, where the surfactant has been removed by heating under vacuum, as expected, bulk-like behavior is observed (not shown). The saturation field is smaller for the 2D self assembly than for the disordered samples. This confirms the magnetic collective behavior of the former in contrast to the behavior of a random collection of magnetic nanoparticles.

In conclusion, in this paper, we report the synthesis of monodispersed single crystal cobalt nanoparticles. The magnetic properties of these samples appear to depend as much on the structure of the particle as on the ordering of those particles, showing a collective, ferromagnetic-like, behavior for the close packed self assembled particles arrays. This behavior is different from that commonly observed in samples with a larger dispersion in sizes, shapes or interparticle distances.

\section{REFERENCES}

[1] G. Shmid and F. L. Chi, "Metals, clusters and colloids," Adv. Mater, vol. 10, pp. 515-526, 1998.

[2] F. J. Himpsel, J. E. Ortega, G. J. Mankey, and R. F. Willis, "Magnetic nanostructures," Adv. in Phys., vol. 47, pp. 511-597, 1998.

[3] D. N. Lambeth, "Hard disk media: Future problems and possible solutions," Vacuum, vol. 59, pp. 522-530, 2000.

[4] S. Sun and C. B. Murray, "Synthesis of monodisperse cobalt nanocrystals and their assembly in magnetic superlattices," J. Appl. Phys., vol. 85, pp. 4325-4330, 1999.

[5] S. Sun, C. B. Murray, D. Weller, L. Folks, and A. Moser, "Monodisperse FePt nanoparticles and feromagnetic FePt nanocrystal superlattices," Science, vol. 287, pp. 1989-1992, 2000.

[6] C. Petit, A. Taleb, and P. J. Pileni, "Co nanosized particles organized in a 2D superlattice: Synthesis, characterization and magnetic properties," Phys. Chem., vol. 103, pp. 1805-1810, 1999.

[7] Z. L. Wang, "Structural analysis of self-assembling nanocrystal superlattices," Adv. Mater, vol. 10, pp. 13-30, 1998.

[8] P. Alivisatos, "Semiconductor clusters, nanocrystals and quantum dots," Science, vol. 271, pp. 933-937, 1996.

[9] V. F. Puntes, P. Alivisatos, and K. M. Krishnan, Appl. Phys. Lett., submitted for publication.

[10] D. Dinega and M. Bawendi, "A solution-phase chemical approach to a new crystal structure of cobalt," Angew. Chem., vol. 38, pp. 1788-1791, 1999 .

[11] S. Chikazumi, Physics of Magnetism: R. E. Krieger Publishing Co., 1964.

[12] D. L. Leslie-Pelecky and R. D. Rieke, "Magnetic properties of nanostructured materials," Chem. Mater, vol. 8, pp. 1770-1783, 1996.

[13] V. Franco, X. Batlle, A. Labarta, and K. O'grady, "Nature of interaction in $\mathrm{CoFeAgCu}$ granular thin films," J. Phys. D., vol. 33, pp. 609-613, 2000.

[14] W. Luo, S. Nagel, T. Rosenbaum, and R. Rosenweig, "Dipole interactions with random anisotropy in a frozen ferrofluid," Phys. Rev. Lett., vol. 67 , pp. 2721-2724, 1991.

[15] J. L. Dorman, D. Fiorani, and E. Tronc, "Magnetic relaxation in small particles systems," Adv. in Chem. Phys., vol. 98, pp. 283-494, 1997. 